\title{
Classification of Mushrooms to Detect their Edibility Based on Key Attributes
}

\author{
V. Vanitha ${ }^{1}$, M.N. Ahil ${ }^{2}$ and N. Rajathi ${ }^{3}$ \\ ${ }^{1}$ Professor, Department of Information Technology, Kumaraguru College of Technology, Coimbatore, India. \\ ${ }^{2}$ Student, Department of Information Technology, Kumaraguru College of Technology, Coimbatore, India. \\ ${ }^{3}$ Professor, Department of Information Technology, Kumaraguru College of Technology, Coimbatore, India.
}

\section{ABSTRACT}

Mushroom is found to be one of the best nutritional foods with high proteins, vitamins and minerals. It contains antioxidants that prevent people from heart disease and cancer. Around 45000 species of mushroom are found to be existing in the world-wide. Among these, only some of the mushroom varieties were found to be edible. Some of them are really dangerous to consume. In order to distinguish between the edible and poisonous mushrooms in the mushroom dataset which was obtained from UCI Machine Learning Repository, some data mining techniques are used. Weka is a data mining tool that has various machine learning algorithms which can be used to pre-process, analyse, classify, visualise and predict the given data. Thus in order to select the attributes that helps in the better classification of mushrooms, Wrapper method and Filter method in Weka are used to identify the best attributes for the classification. The attributes 'odor' and 'spore_print_color' were chosen to be the best ones that contributed to the better classification of edible and poisonous mushrooms. After the identification of the key attributes, classification is performed and decision tree is constructed based on those attributes and its Precision, Recall and F-Measure values are analysed.

KEY WORDS: CLASSIFICATION, FILTER METHOD, KEY ATTRIBUTES, WRAPPER METHOD. .

\section{INTRODUCTION}

Mushroom is considered to be one of the super food sources of vitamins, minerals and several nutrients. Mushrooms are low in calories, they are free of fat, cholesterol and gluten and the sodium levels in mushroom are found to be low. Thus, these are some of the facts that make mushroom to be one of the healthier foods. Mushroom contains Vitamin B, Potassium, Copper, Selenium, Complex carbohydrates and many more beneficial nutrients. Scientists have discovered that

\section{ARTICLE INFORMATION}

${ }^{*}$ Corresponding Author: vanitha.v.cse@kct.ac.in

Received 9th Oct 2020 Accepted after revision 12th Dec 2020

Print ISSN: 0974-6455 Online ISSN: 2321-4007 CODEN: BBRCBA

Thomson Reuters ISI Web of Science Clarivate Analytics USA and Crossref Indexed Journal

\section{Clarivate
Analytics}

NAAS Journal Score 2020 (4.31) SJIF: 2020 (7.728)

A Society of Science and Nature Publication,

Bhopal India 2020. All rights reserved.

Online Contents Available at: http//www.bbrc.in/

Doi: $h$ ttp://dx.doi.org/10.21786/bbrc/13.11/9 the mushrooms help in preventing the breast cancer. Mushrooms contain antioxidants that help in preventing the body cells from getting affected by chronic diseases (Jiang, J et al, 2010). There are almost 45000 species of mushrooms present in the world out of which only some are edible (Husaini, M et al, 2018).

Thus, it is important to classify the mushrooms as edible and poisonous. For classifying the mushrooms as edible and poisonous, a dataset containing 8124 instances and 22 attributes of mushroom was obtained from UCI Machine Learning Repository (Dua, D et al, 2019). Then the dataset is preprocessed and it is analysed using Weka and a decision tree is also constructed for the dataset using data mining techniques. The data mining tool, WEKA (Waikato Environment for Knowledge Analysis), includes a collection of data mining algorithms and also contains options for data preprocessing, clustering, classification, regression, visualisation. 
Data mining is actually a process where it helps in converting a massive amount of data into some useful information (i.e), it helps in predicting the future results with the help of the past acquired results. In (Ismail, S et al, 2018), the paper aims at studying the behavioural features of mushroom which includes surface and shape of the mushroom and mushroom cap's color, features about the gill and stalk of the mushroom, its odor and many other features. Inorder to select the best features, algorithm such as Principal Component Analysis (PCA) is used and for performing the classification, Decision Tree (DT) algorithm is used. The feature, 'odor' was considered to be the highest ranked feature that helped in achieving the high classification accuracy.

In (Pinky, N.J. et al, 2019), Bagging, Boosting and Random Forests are some of the Ensemble methods that have been used to detect whether the mushroom is edible or not. Thus, good results are obtained by using Random Forests for the models that had fixed features for the test sets. In (Eusebi, C et al, 2008), algorithms like unpruned decision tree, voted perceptron algorithm, covering algorithm that generates only correct rules and the nearest neighbor classifier have been used to analyze the mushroom database. Furthermore, (Lavanya, B et al, 2017) suggests that Data mining algorithms such as ID3, CART, and HoeffdingTree (HT) based on decision tree in R studio software environment can be used to find whether the mushroom is edible or not. The paper (M. Senthamilselvi et al, 2018), aims at comparing Weka and Orange tool by analysing Naïve Bayes algorithm which is probability based and decision tree based J48 algorithm in both the tools by using the Mushroom dataset taken from UCI Machine Learning Repository. It is also found that the J48 algorithm was found to produce better results than Naïve Bayes algorithm.

In (Maniraj, V et al, 2015), the classification process is used to categorize whether or not the mushroom is fit for human consumption and clustering is used in the process of identifying the characteristics of the mushroom. Then the association rule is implemented in order to find the best rule so that a decision can be made to check whether the mushroom is edible or not. Thus, it is found that the result obtained by using the decision tree produces the best results of classifying the edible and the poisonous mushrooms. (Wibowo, A et al, 2018) say that mushrooms can be classified into poisonous and edible using machine learning and data mining techniques. The classification algorithms such as Naïve Bayes,

Decision Tree (C4.5) and Support Vector Machine (SVM) have been used for classification and the experiment is performed with the help of Weka. Results imply that the C4.5 algorithm has almost the same accuracy level as that of SVM, but in terms of speed, C4.5 was found to be faster than SVM. Also, Dutta, M in (Verma, S.K. et al, 2018) stated that Artificial Neural Network, Adaptive Neuro Fuzzy inference system and Naïve Bayes are the techniques used for classification to classify the edible and non-edible mushrooms. The performance of ANN and ANFIS are evaluated using accuracy, MAE, kappa statistic. Thus, the performance of ANFIS was found to be more accurate than ANN. ANFIS also had lowest mean absolute error.

In (Mali H. Hakem Alameady*, 2017), Feed-forward Artificial Neural Network (ANN) is used to classify the mushrooms as edible and poisonous. To train the dataset, Multi-Layer Perception is used. It is likewise used to test the data so that it develops a model that helps in predicting the classification. Neural Connection Version 2.0. is a software used for mining the data. This paper aims at explaining Classification, Multi-Layer Preceptor, Back propagation and other mining activities that have been performed on mushroom dataset so that it predict whether the mushroom is fit to consume or not. There are also several datasets that contains the images of the mushroom inspite of the categorical data.

For that in (Ottom et al, 2019), various techniques like Decision Tree, k Nearest Neighbors (KNN), neural network (NN), Support Vector Machines (SVM) are used over a dataset where the dataset contained only the images of the mushroom with background and without background. Thus, an accuracy of $94 \%$ is obtained by using KNN with the help of features that are extracted from the images with real dimensions and for the features which has been obtained from the images, an accuracy of $87 \%$ is obtained. This paper aims at identifying the key attributes of the mushroom that contributed in detecting the edibility of the mushroom. The dataset which was in nominal format was converted into numerical format using Python. And by using the techniques, Wrapper method and Filter method, it has been found that the attributes "odor" and "spore_print_color" contributed to the better classification of the mushrooms inspite of the other attributes.

\section{MATERIAL AND METHODS}

This section is about the methods that have been implemented in the project. The methods included the Data Collection, Pre-processing (Data Transformation), Attribute selection using Wrapper and Filter methods and Decision tree construction using the key attributes.

Data Collection: Since the project is about detecting the edibility of the mushrooms, the dataset containing 8124 instances and 22 attributes of mushroom have been obtained from the UCI Machine Learning Repository (Dua, D et al, 2019). The attributes of the mushroom mentioned in the dataset have been listed in the Figure 1. 
Figure 1: Attributes in the Mushroom Dataset

\begin{tabular}{|c|c|c|c|c|c|c|c|}
\hline capthape & $\begin{array}{c}\text { cap } \\
\text { surface }\end{array}$ & capocolor & brises & odor & $\begin{array}{c}\text { gil- } \\
\text { andment }\end{array}$ & $\begin{array}{l}\text { git. } \\
\text { spacing }\end{array}$ & gill-size \\
\hline gilledor & $\begin{array}{l}\text { stalk. } \\
\text { shape }\end{array}$ & stalk-root & $\begin{array}{l}\text { stal } \\
\text { sufface. } \\
\text { abovering }\end{array}$ & $\begin{array}{l}\text { stilk-suffact } \\
\text { beloning }\end{array}$ & $\begin{array}{l}\text { stik-color- } \\
\text { abvirering }\end{array}$ & $\begin{array}{c}\text { statle- } \\
\text { collor- } \\
\text { beloning }\end{array}$ & ret-lope \\
\hline reil-color & 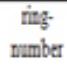 & nisg-type & $\begin{array}{c}\text { sport-priat- } \\
\text { color }\end{array}$ & Poplation & & babitat & \\
\hline
\end{tabular}

Pre-Processing: Pre-Processing is an essential process where the data that has been collected may contain null values, missing values, unwanted data that may lead to the production of wrong results. Hence pre-processing is an essential step where in the irrelevant data can be eliminated so there will be a better accuracy in the final results. Weka has an option for preprocessing under the Explorer interface, in which the file that is needed to be preprocessed is uploaded. Then, the necessary steps on pre-processing are performed. With the obtained results from the pre-processing of the dataset, the attributes that are found to be unwanted are removed. The mushroom dataset which has nominal data is encoded using Python. Then, the encoded data was fed into the Weka tool. It was then analysed for any missing values. For instance, in our mushroom dataset, the attribute "Stalk_root" had around 2480 missing values which will obviously does not help in producing the better results. Hence, those kinds of attributes must be removed.

\section{METHODOLOGY}

Figure 2: Methodology for classification of Mushroom

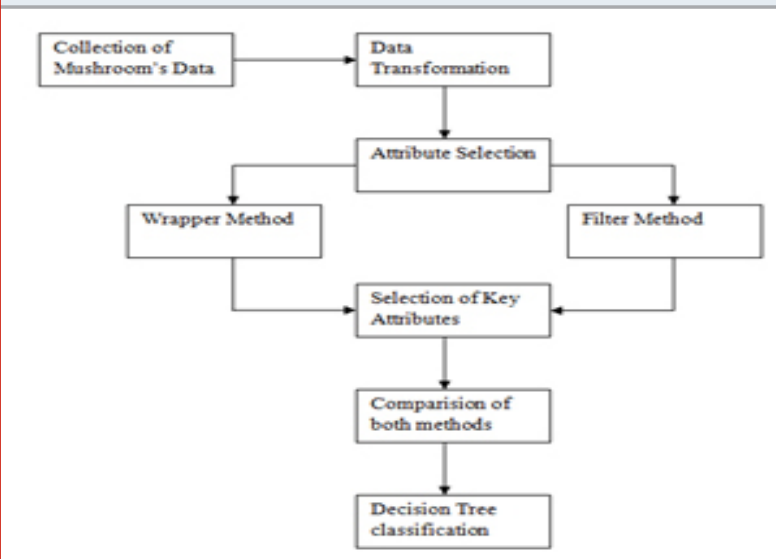

The first step involves uploading the encoded data into the Weka Explorer. Then there is an option called "Select attributes" in the Weka Explorer which has several methods for selecting the highest ranked attributes among a group of attributes. The attribute selection is mainly done to remove the attributes that contributed the least in the classification of mushrooms. It can also be viewed as a method that helps in the dimensionality reduction. Thus in this project, two types of attribute selection have been performed and the results are compared.

Table 1. Key Attributes Selection

\begin{tabular}{|c|c|c|c|}
\hline \multicolumn{4}{|c|}{ KEY ATTRIBUTES SELECTION } \\
\hline \multicolumn{3}{|c|}{ Wrapper Method } & Filter Method \\
\hline \multicolumn{4}{|c|}{ Selected Attributes } \\
\hline $\begin{array}{l}\text { Naïve } \\
\text { Bayes }\end{array}$ & $\mathrm{J} 48$ & Bagging & \multirow{2}{*}{$\begin{array}{l}\text { Ranked } \\
\text { Attributes: } \\
\text { 'Odor' }\end{array}$} \\
\hline \multirow[t]{5}{*}{ 'Odor' } & 'Cap_surface' & 'Cap_surface' & \\
\hline & 'Cap_color' & 'Cap_color' & $\begin{array}{l}\text { 'Spore_print_c } \\
\text { olor' }\end{array}$ \\
\hline & ‘Odor' & 'Odor' & 'Gill_color' \\
\hline & $\begin{array}{l}\text { 'Stalk_surface_ab } \\
\text { ove_ring' }\end{array}$ & $\begin{array}{l}\text { 'Stalk_surface_ab } \\
\text { ove_ring' }\end{array}$ & 'Ring_type' \\
\hline & 'Spore_print_color & 'Spore_print_color & $\begin{array}{l}\text { 'Stalk_surface } \\
\text { above_ring' }\end{array}$ \\
\hline
\end{tabular}

Figure 3: Decision Tree Representation based on Key Attributes

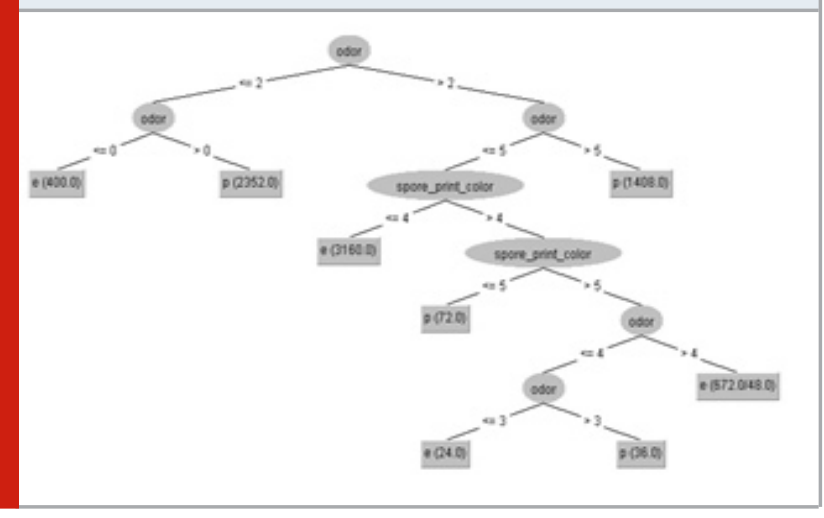

The first method is the Wrapper method which has a classifier subset evaluator that helps in finding all the possible subsets from the given set of attributes. Then the next step includes applying the classifier algorithm like Naïve Bayes, Bagging and J48. After which a search method must be chosen in order to find the best attributes. The search methods include different types like BestFirst, GreedyStepwise and Ranker. In this project, the classifier algorithms Naïve Bayes, Bagging and J48 and the search method BestFirst were used on the dataset and the key attributes were identified. The second method 
that has been used for the attribute selection is the Filter method. The Filter method helps in finding the rank for all the attributes in the dataset out of which one can choose the key attributes based on the rank mentioned for that attribute. In this project, for the filter method, information gain attribute evaluator is used. In order to use the information gain attribute evaluator, rank search method must be used. Then the ranked attributes will appear as an output out of which the top ranked attributes are chosen for classification. The results are tabulated in the below Table I.

Since the attributes "odor" and "spore_print_color" are found to be common in both the attribute selection methods and found to be highly ranked, they are selected as key attributes. Then the selected key attributes are classified under the "Classify" option in the Weka Explorer by using the J48 algorithm and a Decision Tree is constructed based on the key attributes. Its precision, recall and F-measure values are also analysed which were found to be good.
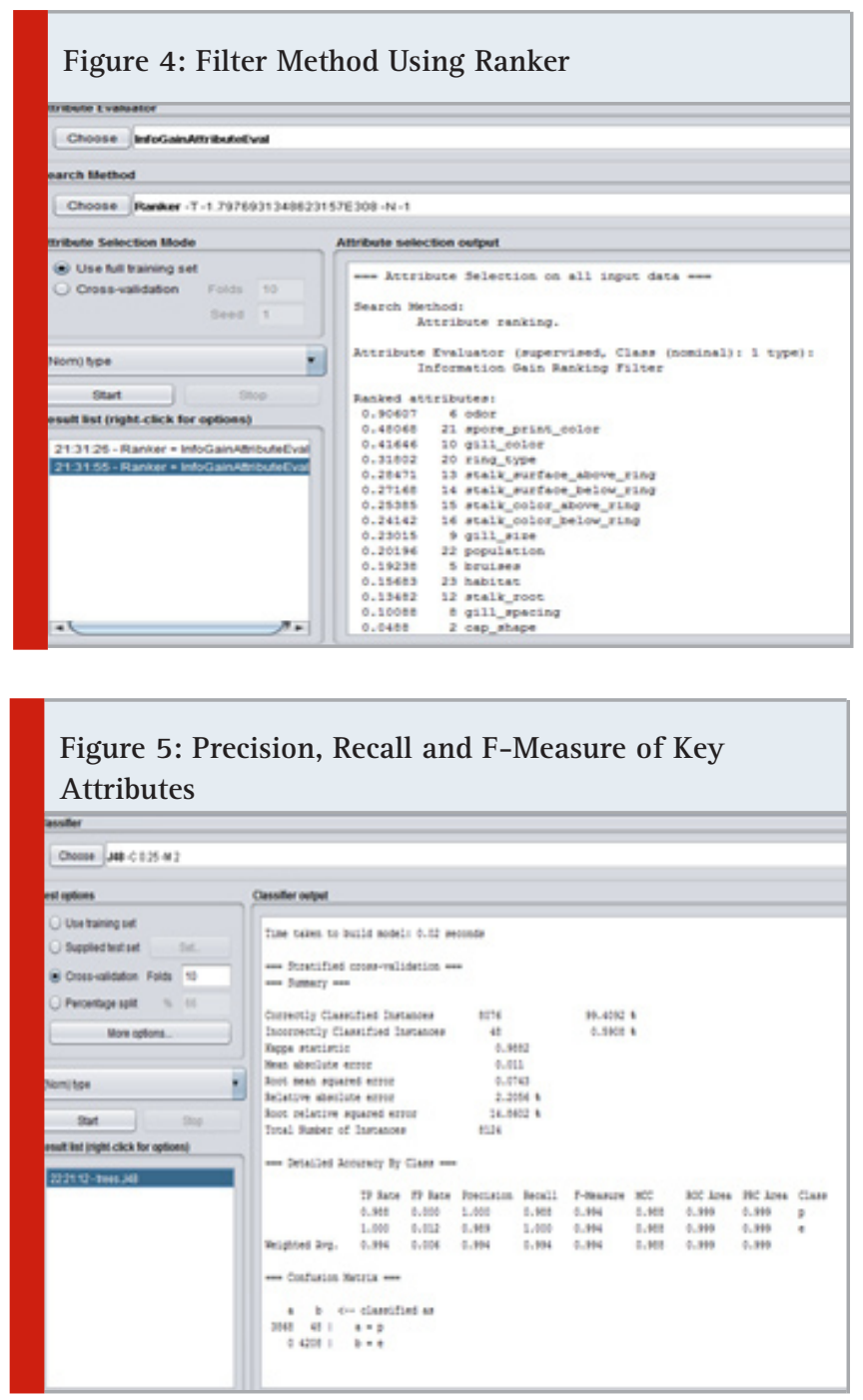

\section{RESULTS AND DISCUSSION}

Thus, by using the Wrapper method and Filter method, the Key Attributes that contributed to the better classification of mushrooms are identified. The attributes that have been found to be the best ones from both the attribute selection methods are compared. It is found that both the attribute selection methods almost gave the same results as the output. Hence by using these attributes as the key attributes, there will be a better accuracy in the classification of mushrooms as edible or poisonous.The key attributes were also found to have good Precision, Recall and F-Measure values.

\section{CONCLUSION}

This paper discussed about the methods of preprocessing, steps to identify the key attributes that helps in the better classification of edible and poisonous mushrooms and also a comparison between the attribute selection methods in order to find whether both the methods produces the same output.

\section{REFERENCES}

Dua, D. and Graff, C. (2019). UCI Machine Learning Repository [http://archive.ics.uci.edu/ml].

Eusebi, C., Gliga, C., John, D. and Maisonave, A., (2008). Data Mining on Mushroom Database. Journal of CSIS, Pace University, pp.1-9.

Husaini, M., (2018). A Data Mining Based On Ensemble Classifier Classification Approach for Edible Mushroom Identification.

Ismail, S., Zainal, A.R. and Mustapha, A., (2018), April. Behavioural features for mushroom classification. In 2018 IEEE Symposium on Computer Applications \& Industrial Electronics (ISCAIE) (pp. 412-415). IEEE.

Jiang, J. and Sliva, D., (2010). Novel medicinal mushroom blend suppresses growth and invasiveness of human breast cancer cells. International journal of oncology, 37(6), pp.1529-1536.

Lavanya, B. and Preethi, G.R., (2017) Performance Analysis of Decision Tree Algorithms on Mushroom Dataset. International Journal for Research in Applied Science and Engineering Technology, 5, pp.183-191.

M. Senthamilselvi, P.S.S. Akilashri, (2018). "A Comparative Study on Weka, Orange Tool for Mushroom Data Set”, International Journal of Computer Sciences and Engineering, Vol.06, Issue.11, pp.231-236, 2018.

Mali H. Hakem Alameady*, (2017). CLASSIFYING POISONOUS AND EDIBLE MUSHROOMS IN THE AGARICUS AND LEPIOTA FAMILY USING MULTILAYER PERCEPTION. INTERNATIONAL JOURNAL OF ENGINEERING SCIENCES \& RESEARCH TECHNOLOGY, 6' (1), pp.154-164. 
Maniraj, V. and Nithya, J., (2015). Integrating Ontology with Data mining with a Case of Mushroom Analysis. Compusoft, 4(10), p.1983.

Ottom, Mohammad Ashraf. (2019). Classification of Mushroom Fungi Using Machine Learning Techniques. International Journal of Advanced Trends in Computer Science and Engineering. 8. 2378-2385. 10.30534/ ijatcse/2019/78852019.

Pinky, N.J., Islam, S.M. and Rafia, S.A., (2019).

Classification Edibility Detection of Mushroom Using
Ensemble Methods. International Journal of Image, Graphics and Signal Processing, 11(4), p.55.

Verma, S.K. and Dutta, M., (2018). Mushroom classification using ANN and ANFIS algorithm. IOSR Journal of Engineering (IOSRJEN), 8(01), pp.94-100. Wibowo, A., Rahayu, Y., Riyanto, A. and Hidayatulloh, T., (2018), March. Classification algorithm for edible mushroom identification. In 2018 International Conference on Information and Communications Technology (ICOIACT) (pp. 250-253). IEEE. 